doi: $10.2306 /$ scienceasia1513-1874.2018.44.054

\title{
A companion of Ostrowski's inequality for complex functions defined on the unit circle and applications
}

\author{
Gang Li, Yue Luan, Jiangyong Yu*
}

College of Mathematics and Statistics, Nanjing University of Information Science and Technology, Nanjing 210044, China

*Corresponding author, e-mail: yjyamxp@outlook.com

Received 3 Jul 2017

Accepted 15 Oct 2017

ABSTRACT: Some companions of Ostrowski's inequality for complex functions defined on the unit circle are proved. Our results in special cases not only recapture known results, but also give a smaller estimator than that of the known results. Applications to a composite quadrature rule and to functions of unitary operators in Hilbert spaces are also considered.

KEYWORDS: Riemann-Stieltjes integral inequalities, unitary operators in Hilbert spaces, quadrature rules

MSC2010: 26D15 41A55

\section{INTRODUCTION}

The Riemann-Stieltjes integral plays an important role in mathematics. In Ref. 1, Alomari used $f(x)\left\{u\left(\frac{1}{2}[a+b]\right)-u(a)\right\}+f(a+b-x)\left\{u(b)-u\left(\frac{1}{2}[a+\right.\right.$ $b])\}$ to approximate the Riemann-Stieltjes integral $\int_{a}^{b} f(t) \mathrm{d} u(t)$ and proved that

$|D(f, u ; a, b, x)| \leqslant H\left[\frac{b-a}{4}+\left|x-\frac{3 a+b}{4}\right|\right]^{r} V_{a}^{b}(u)$,

for any $x \in\left[a, \frac{1}{2}(a+b)\right]$, provided that $f:[a, b] \rightarrow \mathbb{R}$ is an $(r-H)$-Hölder type mapping and $u:[a, b] \rightarrow \mathbb{R}$ is a mapping of bounded variation on $[a, b]$, where $V_{a}^{b}(f)$ denotes the total variation of $f$ on $[a, b]$, and

$$
\begin{gathered}
D(f, u ; a, b, x):=\int_{a}^{b} f(t) \mathrm{d} u(t)-f(x)\left[u\left(\frac{a+b}{2}\right)\right. \\
-u(a)]-f(a+b-x)\left[u(b)-u\left(\frac{a+b}{2}\right)\right]
\end{gathered}
$$

is the error functional, $H>0$ and $r \in(0,1]$ are given.

If the integrand $f:[a, b] \rightarrow \mathbb{R}$ is of bounded variation on $[a, b]$ and the integrator $u(t)=t, t \in$ $[a, b]$, then the following companion of Ostrowski's inequalities for functions of bounded variation has been considered by in Ref. 2, in which they obtained the bound

$$
\begin{aligned}
& \left|\frac{1}{2}[f(x)+f(a+b-x)]-\frac{1}{b-a} \int_{a}^{b} f(t) d t\right| \\
& \leqslant \frac{1}{b-a}\left[(x-a) V_{a}^{x}(f)+\left(\frac{a+b}{2}-x\right) V_{x}^{a+b-x}(f)\right. \\
& \left.+(x-a) V_{a+b-x}^{b}(f)\right] \\
& \leqslant\left\{\begin{array}{l} 
\\
{\left[\frac{1}{4}+\left|\frac{x-\frac{3 a+b}{4}}{b-a}\right|\right] V_{a}^{b}(f), \text { and }} \\
{\left[2\left(\frac{x-a}{b-a}\right)^{\alpha}+\left(\frac{\frac{a+b}{2}-x}{b-a}\right)^{\alpha}\right]^{1 / \alpha}\left[\left[V_{a}^{x}(f)\right]^{\beta}\right.} \\
\left.\quad+\left[V_{x}^{a+b-x}(f)\right]^{\beta}+\left[V_{a+b-x}^{b}(f)\right]^{\beta}\right]^{1 / \beta}, \\
\text { if } \alpha>1, \frac{1}{\alpha}+\frac{1}{\beta}=1, \text { and } \\
\frac{x-a+\frac{b-a}{2}}{b-a} \max \left\{V_{a}^{x}(f), V_{x}^{a+b-x}(f),\right. \\
\left.V_{a+b-x}^{b}(f)\right\},
\end{array}\right.
\end{aligned}
$$

for any $x \in\left[a, \frac{1}{2}(a+b)\right]$.

In Ref. 3, Dragomir developed Ostrowski's type integral inequality for the complex unit circle $\mathbb{C}(0,1)$.

Theorem 1 Assume that $f: C(0,1) \rightarrow \mathbb{C}$ satisfies the following Hölder's type condition

$$
|f(a)-f(b)| \leqslant H|a-b|^{r},
$$

for any $a, b \in C(0,1)$, where $H>0$ and $r \in(0,1]$ are given. If $[a, b] \subseteq[0,2 \pi]$ and the function $u:[a, b] \rightarrow$ 
$\mathbb{C}$ is of bounded variation, then

$$
\begin{aligned}
\mid f\left(\mathrm{e}^{\mathrm{i} s}\right)[u(b) & -u(a)]-\int_{a}^{b} f\left(\mathrm{e}^{\mathrm{i} t}\right) \mathrm{d} u(t) \mid \\
& \leqslant 2^{r} H \max _{t \in[a, b]}\left|\sin ^{r}\left(\frac{s-t}{2}\right)\right| V_{a}^{b}(u),
\end{aligned}
$$

for any $s \in[a, b]$.

For other inequalities for the Riemann-Stieltjes integral, see Refs. 4-19.

Motivated by the above facts, we consider in the present paper the problem of approximating the Riemann-Stieltjes integral $\int_{a}^{b} f\left(\mathrm{e}^{\mathrm{i} s}\right) \mathrm{d} u(s)$ by the rule

$$
\begin{aligned}
& f\left(\mathrm{e}^{\mathrm{i} s}\right)\left[u\left(\frac{a+b}{2}\right)-u(a)\right] \\
& \quad+f\left(\mathrm{e}^{\mathrm{i}(a+b-s)}\right)\left[u(b)-u\left(\frac{a+b}{2}\right)\right],
\end{aligned}
$$

where the continuous complex valued function $f$ : $C(0,1) \rightarrow \mathbb{C}$ is defined on the complex unit circle $C(0,1)$ and the function $u:[a, b] \subseteq[0,2 \pi] \rightarrow \mathbb{C}$ is of bounded variation.

We denote the error functional

$$
\begin{aligned}
& T(f, u ; a, b ; s, t):=f\left(\mathrm{e}^{\mathrm{i} s}\right)\left[u\left(\frac{a+b}{2}\right)-u(a)\right] \\
& +f\left(\mathrm{e}^{\mathrm{i}(a+b-s)}\right)\left[u(b)-u\left(\frac{a+b}{2}\right)\right]-\int_{a}^{b} f\left(\mathrm{e}^{\mathrm{i} t}\right) \mathrm{d} u(t),
\end{aligned}
$$

where $t \in[a, b]$ and $f$ is of $(r-H)$-Hölder type and $u$ is the function of bounded variation.

The outline of this paper is as follows. First, we show some inequalities for the Riemann-Stieltjes integral. Second, we apply them to a composite quadrature rule. Third, the study of applications to unitary operators is discussed.

\section{SOME COMPANIONS OF OSTROWSKI'S TYPE INEQUALITY}

The following companions of Ostrowski's inequality for Riemann-Stieltjes integrals hold.

Theorem 2 Suppose that $f: C(0,1) \rightarrow \mathbb{C}$ satisfies the following Hölder's type condition:

$$
|f(a)-f(b)| \leqslant H|a-b|^{r},
$$

for any $a, b \in C(0,1)$, where $H>0$ and $r \in(0,1]$ are given. If $[a, b] \subseteq[0,2 \pi]$ and $u:[a, b] \rightarrow \mathbb{C}$ is the function of bounded variation, then

$$
f\left(\mathrm{e}^{\mathrm{is} s}\right)\left[u\left(\frac{a+b}{2}\right)-u(a)\right]-\int_{a}^{b} f\left(\mathrm{e}^{\mathrm{i} t}\right) \mathrm{d} u(t)
$$

$$
\begin{gathered}
+f\left(\mathrm{e}^{\mathrm{i}(a+b-s)}\right)\left[u(b)-u\left(\frac{a+b}{2}\right)\right] \mid \\
\leqslant 2^{r} H\left\{\max _{t \in[a,((a+b) / 2)]}\left|\sin \left(\frac{s-t}{2}\right)\right|^{r} V_{a}^{(a+b) / 2}(u)\right. \\
\left.\quad+\max _{t \in[(a+b) / 2, b]}\left|\sin \left(\frac{a+b-s-t}{2}\right)\right|^{r} V_{(a+b) / 2}^{b}(u)\right\} \\
\leqslant 2^{r} H \sin ^{r}\left[\frac{b-a}{8}+\frac{1}{2}\left|s-\frac{3 a+b}{4}\right|\right] V_{a}^{b}(u),
\end{gathered}
$$

for any $s \in\left[a, \frac{1}{2}(a+b)\right]$.

Proof: Clearly, we have from Ref. 1

$$
\begin{array}{r}
f\left(\mathrm{e}^{\mathrm{i} s}\right)\left[u\left(\frac{a+b}{2}\right)-u(a)\right]-\int_{a}^{b} f\left(\mathrm{e}^{\mathrm{i} t}\right) \mathrm{d} u(t) \\
+f\left(\mathrm{e}^{\mathrm{i}(a+b-s)}\right)\left[u(b)-u\left(\frac{a+b}{2}\right)\right] \\
=\int_{(a+b) / 2}^{b}\left[f\left(\mathrm{e}^{\mathrm{i}(a+b-s)}\right)-f\left(\mathrm{e}^{\mathrm{i} t}\right)\right] \mathrm{d} u(t) \\
\quad+\int_{a}^{(a+b) / 2}\left[f\left(\mathrm{e}^{\mathrm{i} s}\right)-f\left(\mathrm{e}^{\mathrm{i} t}\right)\right] \mathrm{d} u(t),
\end{array}
$$

for any $s \in\left[a, \frac{1}{2}(a+b)\right]$.

If $q:[c, d] \rightarrow \mathbb{C}$ is a continuous function and $v:[c, d] \rightarrow \mathbb{C}$ is the function of bounded variation, then there exists the Riemann-Stieltjes integral $\int_{c}^{d} q(t) \mathrm{d} v(t)$ and

$$
\left|\int_{c}^{d} q(t) \mathrm{d} v(t)\right| \leqslant \max _{t \in[c, d]}|q(t)| V_{c}^{d}(v) .
$$

Applying inequality (9) to identity (8) and using Hölder's type condition (6), we obtain

$$
\begin{aligned}
& \mid f\left(\mathrm{e}^{\mathrm{i} s}\right)\left[u\left(\frac{a+b}{2}\right)-u(a)\right]-\int_{a}^{b} f\left(\mathrm{e}^{\mathrm{i} t}\right) \mathrm{d} u(t) \\
& \quad+f\left(\mathrm{e}^{\mathrm{i}(a+b-s)}\right)\left[u(b)-u\left(\frac{a+b}{2}\right)\right] \mid \\
& \leqslant \max _{t \in[a,((a+b) / 2)]}\left|f\left(\mathrm{e}^{\mathrm{i} s}\right)-f\left(\mathrm{e}^{\mathrm{i} t}\right)\right| V_{a}^{(a+b) / 2}(u) \\
&+\max _{t \in[(a+b) / 2, b]}\left|f\left(\mathrm{e}^{\mathrm{i}(a+b-s)}\right)-f\left(\mathrm{e}^{\mathrm{i} t}\right)\right| V_{(a+b) / 2}^{b}(u) \\
& \leqslant H\left\{\max _{t \in[a,((a+b) / 2)]}\left|\mathrm{e}^{\mathrm{i} s}-\mathrm{e}^{\mathrm{i} t}\right|^{r} V_{a}^{a+b}(u)\right. \\
&\left.+\max _{t \in[(a+b) / 2, b]}\left|\mathrm{e}^{\mathrm{i}(a+b-s)}-\mathrm{e}^{\mathrm{i} t}\right|^{r} V_{(a+b) / 2}^{b}(u)\right\} .
\end{aligned}
$$

From Ref. 3, we have

$$
\left|\mathrm{e}^{\mathrm{is}}-\mathrm{e}^{\mathrm{i} t}\right|^{r}=2^{r}\left|\sin \left(\frac{s-t}{2}\right)\right|^{r},
$$


for any $s, t \in \mathbb{R}$.

Applying (11) to (10), we deduce

$$
\begin{aligned}
\mid f\left(\mathrm{e}^{\mathrm{i} s}\right)\left[u\left(\frac{a+b}{2}\right)-u(a)\right]-\int_{a}^{b} f\left(\mathrm{e}^{\mathrm{i} t}\right) \mathrm{d} u(t) \\
+f\left(\mathrm{e}^{\mathrm{i}(a+b-s)}\right)\left[u(b)-u\left(\frac{a+b}{2}\right)\right] \mid \\
\leqslant 2^{r} H\left\{\max _{t \in[a,((a+b) / 2)]}\left|\sin \left(\frac{s-t}{2}\right)\right|^{r} V_{a}^{(a+b) / 2}(u)\right. \\
\left.\quad+\max _{t \in[(a+b) / 2, b]}\left|\sin \left(\frac{a+b-s-t}{2}\right)\right|^{r} V_{(a+b) / 2}^{b}(u)\right\},
\end{aligned}
$$

for any $s \in\left[a, \frac{1}{2}(a+b)\right]$.

We now prove

$$
\begin{aligned}
& 2^{r} H\left\{\max _{t \in[a,((a+b) / 2)]}\left|\sin \left(\frac{s-t}{2}\right)\right|^{r} V_{a}^{(a+b) / 2}(u)+\right. \\
& \left.+\max _{t \in[(a+b) / 2, b]}\left|\sin \left(\frac{a+b-s-t}{2}\right)\right|^{r} V_{(a+b) / 2}^{b}(u)\right\} \\
& \leqslant 2^{r} H \sin ^{r}\left[\frac{b-a}{8}+\frac{1}{2}\left|s-\frac{3 a+b}{4}\right|\right] V_{a}^{b}(u),
\end{aligned}
$$

for any $s \in\left[a, \frac{1}{2}(a+b)\right]$.

By $[a, b] \subset[0,2 \pi]$ and $s \in\left[a, \frac{1}{2}(a+b)\right]$, we have $a+b-s \in\left[\frac{1}{2}(a+b), b\right]$. When $t \in\left[a, \frac{1}{2}(a+b)\right]$, we have $\frac{1}{2}|t-s| \leqslant \frac{1}{2}\left|\frac{1}{2}(a+b)-a\right| \leqslant \frac{1}{2} \pi$. When $t \in\left[\frac{1}{2}(a+\right.$ $b), b]$, we have $\frac{1}{2}|a+b-s-t| \leqslant \frac{1}{2}\left|b-\frac{1}{2}(a+b)\right| \leqslant \frac{1}{2} \pi$. Since the function $\sin (x)$ is monotone increasing on $\left[0, \frac{1}{2} \pi\right]$, we have that

$$
\begin{aligned}
\max _{t \in} & {[a,((a+b) / 2)] } \\
& =\sin \left(\max _{t \in[a,((a+b) / 2)]} \frac{1}{2}|t-s|\right) \\
& =\sin \left(\frac{1}{2} \max \left\{\frac{a+b}{2}-s, s-a\right\}\right) \\
& =\sin \left(\frac{b-a}{8}+\frac{1}{2}\left|s-\frac{3 a+b}{4}\right|\right),
\end{aligned}
$$

for any $s \in\left[a, \frac{1}{2}(a+b)\right]$. In a similar way, we obtain

$$
\begin{aligned}
\max _{t \in[(a+b) / 2, b]} \mid & \sin \left(\frac{a+b-s-t}{2}\right) \mid \\
& =\sin \left(\frac{b-a}{8}+\frac{1}{2}\left|s-\frac{3 a+b}{4}\right|\right),
\end{aligned}
$$

for any $s \in\left[a, \frac{1}{2}(a+b)\right]$. Hence we deduce the desired result (13). If we choose $s=\frac{1}{2}(a+b)$ in
(7), we recapture Remark 2 of Ref. 3. In particular, if we choose $s=(3 a+b) / 4$ in (7), then we obtain

$$
\begin{gathered}
\mid f\left(\mathrm{e}^{\mathrm{i}((3 a+b) / 4)}\right)\left[u\left(\frac{a+b}{2}\right)-u(a)\right]-\int_{a}^{b} f\left(\mathrm{e}^{\mathrm{i} t}\right) \mathrm{d} u(t) \\
+f\left(\mathrm{e}^{\mathrm{i}((a+3 b) / 4)}\right)\left[u(b)-u\left(\frac{a+b}{2}\right)\right] \mid \\
\leqslant 2^{r} H \sin ^{r}\left(\frac{b-a}{8}\right) V_{a}^{b}(u),
\end{gathered}
$$

which is more precise than Remark 2 of Ref. 3.

We can consider the following situation: for any $w, z \in C(0,1)$, the Lipschitz condition $|f(z)-f(w)| \leqslant$ $L|z-w|$ is satisfied, where $f: C(0,1) \rightarrow \mathbb{C}$ and $L>$ 0 . In this case, we can show the sharpness of the corresponding version of (17).

Corollary 1 Suppose that $f: C(0,1) \rightarrow \mathbb{C}$ is Lipschitzian with the constant $L>0$ on the circle $C(0,1)$. If $[a, b] \subset[0,2 \pi]$ and $u:[a, b] \rightarrow \mathbb{C}$ is a function of bounded variation on $[a, b]$, then we have

$$
\begin{gathered}
\mid f\left(\mathrm{e}^{\mathrm{i}((3 a+b) / 4)}\right)\left[u\left(\frac{a+b}{2}\right)-u(a)\right]-\int_{a}^{b} f\left(\mathrm{e}^{\mathrm{i} t}\right) \mathrm{d} u(t) \\
+f\left(\mathrm{e}^{\mathrm{i}((a+3 b) / 4)}\right)\left[u(b)-u\left(\frac{a+b}{2}\right)\right] \mid \\
\leqslant 2 L \sin \left(\frac{b-a}{8}\right) V_{a}^{b}(u), \quad(17)
\end{gathered}
$$

where the constant 2 on the right-hand side cannot be replaced by a smaller constant.

Proof: We only need to prove the sharpness of the constant 2. Assume that (17) holds with a constant $C>0$, that is,

$$
\begin{gathered}
\mid f\left(\mathrm{e}^{\mathrm{i}((3 a+b) / 4)}\right)\left[u\left(\frac{a+b}{2}\right)-u(a)\right]-\int_{a}^{b} f\left(\mathrm{e}^{\mathrm{i} t}\right) \mathrm{d} u(t) \\
+f\left(\mathrm{e}^{\mathrm{i}((a+3 b) / 4)}\right)\left[u(b)-u\left(\frac{a+b}{2}\right)\right] \mid \\
\leqslant C L \sin \left(\frac{b-a}{8}\right) V_{a}^{b}(u) .
\end{gathered}
$$

Choose $f: \mathbb{C} \rightarrow \mathbb{C}, f(z)=z$ and $u:[0,2 \pi] \rightarrow \mathbb{R}$ given by

$$
u(t):= \begin{cases}0, & 0 \leqslant t<2 \pi \\ 1, & t=2 \pi\end{cases}
$$

Clearly, $f$ is Lipschitzian with the constant $L=1$. At the same time, we consider $a=0$ and $b=2 \pi$. By 
using integration by parts for the integral, we have

$$
\begin{aligned}
\int_{0}^{2 \pi} \mathrm{e}^{\mathrm{i} t} \mathrm{~d} u(t) & =\left.\mathrm{e}^{\mathrm{i} t} u(t)\right|_{0} ^{2 \pi}-\mathrm{i} \int_{0}^{2 \pi} \mathrm{e}^{\mathrm{i} t} u(t) \mathrm{d} t \\
& =u(2 \pi)-u(0)-\mathrm{i} \int_{0}^{2 \pi} \mathrm{e}^{\mathrm{i} t} u(t) \mathrm{d} t
\end{aligned}
$$

and

$$
V_{0}^{2 \pi}(u)=1
$$

Consequently, by (20) and (21), we obtain $C \geqslant$ 2.

Remark 1 Under the assumption Theorem 2 and $u(t)=t, t \in[a, b] \subseteq[0,2 \pi]$, we have

$$
\begin{aligned}
\mid f\left(\mathrm{e}^{\mathrm{i} s}\right)+ & f\left(\mathrm{e}^{\mathrm{i}(a+b-s)}\right)-\frac{2}{b-a} \int_{a}^{b} f\left(\mathrm{e}^{\mathrm{i} t}\right) \mathrm{d} t \mid \\
& \leqslant 2^{r} H\left\{\max _{t \in[a,((a+b) / 2)]}\left|\sin \left(\frac{s-t}{2}\right)\right|^{r}\right. \\
& \left.+\max _{t \in[(a+b) / 2, b]}\left|\sin \left(\frac{a+b-s-t}{2}\right)\right|^{r}\right\},
\end{aligned}
$$

for any $s \in\left[a, \frac{1}{2}(a+b)\right]$, provided that $f: C(0,1) \rightarrow$ $\mathbb{C}$ satisfies Hölder's type condition (6).

Remark 2 If $f: C(0,1) \rightarrow \mathbb{C}$ satisfies (6), and $p:[a, b] \subseteq[0,2 \pi] \rightarrow \mathbb{C}$ is Lebesgue integrable on $[a, b]$, then we have

$$
\begin{array}{r}
\mid f\left(\mathrm{e}^{\mathrm{i} s}\right) \int_{a}^{(a+b) / 2} p(t) \mathrm{d} t+f\left(\mathrm{e}^{\mathrm{i}(a+b-s)}\right) \int_{(a+b) / 2}^{b} p(t) \mathrm{d} t \\
-\int_{a}^{b} f\left(\mathrm{e}^{\mathrm{i} t}\right) p(t) \mathrm{d} t \mid \\
\leqslant 2^{r} H\left\{\max _{t \in[a,((a+b) / 2)]}\left|\sin \left(\frac{s-t}{2}\right)\right|^{r} \int_{a}^{(a+b) / 2}|p(t)| \mathrm{d} t\right. \\
\left.+\underset{t \in[(a+b) / 2, b]}{\max }\left|\sin \left(\frac{a+b-s-t}{2}\right)\right|^{r}|p(t)| \mathrm{d} t\right\} .
\end{array}
$$

Theorem 3 On the circle $C(0,1)$, suppose that $f$ : $C(0,1) \rightarrow \mathbb{C}$ is Lipschitzian with the constant $L>0$. If $[a, b] \subseteq[0,2 \pi]$ and the function $u:[a, b] \rightarrow \mathbb{C}$ is
Lipschitzian with the constant $K>0$ on $[a, b]$, then

$$
\begin{array}{r}
\mid f\left(\mathrm{e}^{\mathrm{i} s}\right)\left[u\left(\frac{a+b}{2}\right)-u(a)\right]-\int_{a}^{b} f\left(\mathrm{e}^{\mathrm{i} t}\right) \mathrm{d} u(t) \\
+f\left(\mathrm{e}^{\mathrm{i}(a+b-s)}\right)\left[u(b)-u\left(\frac{a+b}{2}\right)\right] \mid \\
\leqslant 32 K L \sin ^{2}\left(\frac{b-a}{8}\right),
\end{array}
$$

for any $s \in\left[a, \frac{1}{2}(a+b)\right]$.

Proof: If $w:[a, b] \rightarrow \mathbb{C}$ is a Riemann integrable function and the function $v:[a, b] \rightarrow \mathbb{C}$ is $M$ Lipschitzian, then there exists the Riemann-Stieltjes integral $\int_{a}^{b} w(t) \mathrm{d} v(t)$ and

$$
\left|\int_{a}^{b} w(t) \mathrm{d} v(t)\right| \leqslant M \int_{a}^{b}|w(t)| \mathrm{d} t .
$$

Making use of (25), we have from (8) that

$$
\begin{array}{r}
\mid f\left(\mathrm{e}^{\mathrm{i} s}\right)\left[u\left(\frac{a+b}{2}\right)-u(a)\right]-\int_{a}^{b} f\left(\mathrm{e}^{\mathrm{i} t}\right) \mathrm{d} u(t) \\
+f\left(\mathrm{e}^{\mathrm{i}(a+b-s)}\right)\left[u(b)-u\left(\frac{a+b}{2}\right)\right] \mid \\
\leqslant K L\left\{\int_{a}^{(a+b) / 2}\left|\mathrm{e}^{\mathrm{i} s}-\mathrm{e}^{\mathrm{i} t}\right| \mathrm{d} t+\right. \\
\left.\int_{(a+b) / 2}^{b}\left|\mathrm{e}^{\mathrm{i}(a+b-s)}-\mathrm{e}^{\mathrm{i} t}\right| \mathrm{d} t\right\},
\end{array}
$$

for any $s \in\left[a, \frac{1}{2}(a+b)\right]$. By (11), for any $t, s \in \mathbb{R}$, we have $\left|\mathrm{e}^{\mathrm{i} s}-\mathrm{e}^{\mathrm{i} t}\right|=2\left|\sin \left(\frac{1}{2}(s-t)\right)\right|$. Then

$$
\begin{aligned}
\int_{a}^{(a+b) / 2}\left|\mathrm{e}^{\mathrm{i} s}-\mathrm{e}^{\mathrm{it}}\right| \mathrm{d} t & =8\left[\sin ^{2}\left(\frac{s-a}{4}\right)+\sin ^{2}\left(\frac{\frac{a+b}{2}-s}{4}\right)\right] \\
& \leqslant 16 \sin ^{2}\left(\frac{b-a}{8}\right)
\end{aligned}
$$

for any $s \in\left[a, \frac{1}{2}(a+b)\right]$. In the similar way, we have

$$
\int_{(a+b) / 2}^{b}\left|\mathrm{e}^{\mathrm{i}(a+b-s)}-\mathrm{e}^{\mathrm{i} t}\right| \mathrm{d} t \leqslant 16 \sin ^{2}\left(\frac{b-a}{8}\right),
$$

for any $s \in\left[a, \frac{1}{2}(a+b)\right]$. Plugging (27) and (28) into (26), we complete the proof of (24). 
Remark 3 Under the assumptions in Theorem 3,

$$
\begin{gathered}
\mid f\left(\mathrm{e}^{\mathrm{i}((3 a+b) / 4)}\right)\left[u\left(\frac{a+b}{2}\right)-u(a)\right]-\int_{a}^{b} f\left(\mathrm{e}^{\mathrm{i} t}\right) \mathrm{d} u(t) \\
+f\left(\mathrm{e}^{\mathrm{i}((3 b+a) / 4)}\right)\left[u(b)-u\left(\frac{a+b}{2}\right)\right] \mid \\
\leqslant 32 K L \sin ^{2}\left(\frac{b-a}{16}\right) .
\end{gathered}
$$

Remark 4 If $u(t)=t, t \in[a, b]$, then

$$
\begin{aligned}
& \left|f\left(\mathrm{e}^{\mathrm{i} s}\right)+f\left(\mathrm{e}^{\mathrm{i}(a+b-s)}\right)-\frac{2}{b-a} \int_{a}^{b} f\left(\mathrm{e}^{\mathrm{i} t}\right) \mathrm{d} t\right| \\
& \quad \leqslant \frac{32 L}{b-a}\left[\sin ^{2}\left(\frac{s-a}{4}\right)+\sin ^{2}\left(\frac{\frac{a+b}{2}-s}{4}\right)\right] \\
& \quad \leqslant \frac{64 L}{b-a} \sin ^{2}\left(\frac{b-a}{8}\right)
\end{aligned}
$$

for any $s \in\left[a, \frac{1}{2}(a+b)\right]$ and

$$
\begin{array}{r}
\left|f\left(\mathrm{e}^{\mathrm{i}((3 a+b) / 4)}\right)+f\left(\mathrm{e}^{\mathrm{i}((3 b+a) / 4)}\right)-\frac{2}{b-a} \int_{a}^{b} f\left(\mathrm{e}^{\mathrm{i} t}\right) \mathrm{d} t\right| \\
\leqslant \frac{64 L}{b-a} \sin ^{2}\left(\frac{b-a}{16}\right) .
\end{array}
$$

Remark 5 If $w:[a, b] \subseteq[0,2 \pi] \rightarrow \mathbb{C}$ is essentially bounded on $[a, b]$ and $f: C(0,1) \rightarrow \mathbb{C}$ is Lipschitzian with the constant $L>0$ on the circle $C(0,1)$, then for any $s \in\left[a, \frac{1}{2}(a+b)\right]$,

$$
\begin{array}{r}
\mid f\left(\mathrm{e}^{\mathrm{i} s}\right) \int_{a}^{(a+b) / 2} w(t) \mathrm{d} t-\int_{a}^{b} f\left(\mathrm{e}^{\mathrm{i} t}\right) w(t) \mathrm{d} t \\
\quad+f\left(\mathrm{e}^{\mathrm{i}(a+b-s)}\right) \int_{(a+b) / 2}^{b} w(t) \mathrm{d} t \mid \\
\quad \leqslant 32 L\|w\|_{\infty} \sin ^{2}\left(\frac{b-a}{8}\right),
\end{array}
$$

where $\|w\|_{\infty}:=\operatorname{esssup}_{t \in[a, b]}|w(t)|$.

Theorem 4 On the circle $C(0,1)$, suppose that $f$ : $C(0,1) \rightarrow \mathbb{C}$ is Lipschitzian with the constant $L>0$. If $u:[a, b] \subseteq[0,2 \pi]$ and the function $u:[a, b] \rightarrow \mathbb{R}$ is monotonic nondecreasing on $[a, b]$, then for any $s \in\left[a, \frac{1}{2}(a+b)\right]$,

$$
\begin{array}{r}
\mid f\left(\mathrm{e}^{\mathrm{i} s}\right)\left[u\left(\frac{a+b}{2}\right)-u(a)\right]-\int_{a}^{b} f\left(\mathrm{e}^{\mathrm{i} t}\right) \mathrm{d} u(t) \\
+f\left(\mathrm{e}^{\mathrm{i}(a+b-s)}\right)\left[u(b)-u\left(\frac{a+b}{2}\right)\right] \mid
\end{array}
$$

$$
\begin{gathered}
\leqslant L \int_{a}^{(a+b) / 2} \operatorname{sgn}(s-t) \cos \left(\frac{s-t}{2}\right) u(t) \mathrm{d} t \\
+L \int_{(a+b) / 2}^{b} \operatorname{sgn}(a+b-s-t) \cos \left(\frac{a+b-s-t}{2}\right) u(t) \mathrm{d} t \\
+2 L \sin \left(\frac{s-a}{2}\right)[u(b)-u(a)] . \quad(33)
\end{gathered}
$$

Proof: If $p:[a, b] \rightarrow \mathbb{C}$ is a continuous function and $v:[a, b] \rightarrow \mathbb{R}$ is monotonic nondecreasing on $[a, b]$, then there exists the Riemann-Stieltjes integral $\int_{a}^{b} p(t) \mathrm{d} v(t)$ and

$$
\left|\int_{a}^{b} p(t) \mathrm{d} v(t)\right| \leqslant \int_{a}^{b}|p(t)| \mathrm{d} v(t) .
$$

Making use of (34), we have from (8) that

$$
\begin{array}{r}
\mid f\left(\mathrm{e}^{\mathrm{i} s}\right)\left[u\left(\frac{a+b}{2}\right)-u(a)\right]-\int_{a}^{b} f\left(\mathrm{e}^{\mathrm{i} t}\right) \mathrm{d} u(t) \\
+f\left(\mathrm{e}^{\mathrm{i}(a+b-s)}\right)\left[u(b)-u\left(\frac{a+b}{2}\right)\right] \mid \\
\leqslant \int_{(a+b) / 2}^{b}\left|f\left(\mathrm{e}^{\mathrm{i}(a+b-s)}\right)-f\left(\mathrm{e}^{\mathrm{i} t}\right)\right| \mathrm{d} u(t) \\
+\int_{a}^{(a+b) / 2}\left|f\left(\mathrm{e}^{\mathrm{i} s}\right)-f\left(\mathrm{e}^{\mathrm{i} t}\right)\right| \mathrm{d} u(t) \\
\leqslant L \int_{(a+b) / 2}^{b}\left|\mathrm{e}^{\mathrm{i}(a+b-s)}-\mathrm{e}^{\mathrm{i} t}\right| \mathrm{d} u(t) \\
+L \int_{a}^{(a+b) / 2}\left|\mathrm{e}^{\mathrm{i} s}-\mathrm{e}^{\mathrm{i} t}\right| \mathrm{d} u(t),
\end{array}
$$

for any $s \in\left[a, \frac{1}{2}(a+b)\right]$. By (11), for any $t, s \in \mathbb{R}$, we have $\left|\mathrm{e}^{\mathrm{i} s}-\mathrm{e}^{\mathrm{i} t}\right|=2\left|\sin \left(\frac{1}{2}(s-t)\right)\right|$. Then

$$
\begin{aligned}
& \int_{a}^{(a+b) / 2}\left|\mathrm{e}^{\mathrm{i} s}-\mathrm{e}^{\mathrm{i} t}\right| \mathrm{d} u(t) \\
& =2 \int_{a}^{s} \sin \left(\frac{s-t}{2}\right) \mathrm{d} u(t)+2 \int_{s}^{(a+b) / 2} \sin \left(\frac{t-s}{2}\right) \mathrm{d} u(t),
\end{aligned}
$$

for any $s \in\left[a, \frac{1}{2}(a+b)\right] \subseteq[0,2 \pi]$. Using integration by parts for the Riemann-Stieltjes integral, we have

$$
\begin{aligned}
& \int_{a}^{s} \sin \left(\frac{s-t}{2}\right) \mathrm{d} u(t) \\
& \quad=-\sin \left(\frac{s-a}{2}\right) u(a)+\frac{1}{2} \int_{a}^{s} \cos \left(\frac{s-t}{2}\right) u(t) \mathrm{d} t
\end{aligned}
$$


and

$$
\begin{array}{r}
\int_{s}^{(a+b) / 2} \sin \left(\frac{t-s}{2}\right) \mathrm{d} u(t)=\sin \left(\frac{\frac{a+b}{2}-s}{2}\right) u\left(\frac{a+b}{2}\right) \\
-\frac{1}{2} \int_{s}^{(a+b) / 2} \cos \left(\frac{t-s}{2}\right) u(t) \mathrm{d} t . \quad
\end{array}
$$

Hence, by (36), (37) and (38), we have

$$
\begin{aligned}
& \int_{a}^{(a+b) / 2}\left|\mathrm{e}^{\mathrm{i} s}-\mathrm{e}^{\mathrm{i} t}\right| \mathrm{d} u(t) \\
& =2\left[\sin \left(\frac{\frac{a+b}{2}-s}{2}\right) u\left(\frac{a+b}{2}\right)-\sin \left(\frac{s-a}{2}\right) u(a)\right] \\
& \quad+\int_{a}^{(a+b) / 2} \operatorname{sgn}(s-t) \cos \left(\frac{s-t}{2}\right) u(t) \mathrm{d} t .
\end{aligned}
$$

In the similar way, we have

$$
\begin{aligned}
& \int_{(a+b) / 2}^{b}\left|\mathrm{e}^{\mathrm{i}(a+b-s)}-\mathrm{e}^{\mathrm{i} t}\right| \mathrm{d} u(t) \\
& \quad=2\left[\sin \left(\frac{s-a}{2}\right) u(b)-\sin \left(\frac{\frac{a+b}{2}-s}{2}\right) u\left(\frac{a+b}{2}\right)\right] \\
& +\int_{(a+b) / 2}^{b} \operatorname{sgn}(a+b-s-t) \cos \left(\frac{a+b-s-t}{2}\right) u(t) \mathrm{d} t .
\end{aligned}
$$

By (35), (39) and (40), (33) is proved.

Remark 6 If we choose $s=\frac{1}{2}(a+b)$ in (33), we recapture Theorem 5 of Ref. 3 .

Corollary 2 Under the assumptions of Theorem 4, for any $s \in\left[a, \frac{1}{2}(a+b)\right]$,

$$
\begin{aligned}
& \mid f\left(\mathrm{e}^{\mathrm{i} s}\right)\left[u\left(\frac{a+b}{2}\right)-u(a)\right]-\int_{a}^{b} f\left(\mathrm{e}^{\mathrm{i} t}\right) \mathrm{d} u(t) \\
& +f\left(\mathrm{e}^{\mathrm{i}(a+b-s)}\right)\left[u(b)-u\left(\frac{a+b}{2}\right)\right] \mid \\
& \leqslant 2 L \sin \left(\frac{s-a}{2}\right)[u(b)-u(a)+u(s)-u(a+b-s)] \\
& +2 L \sin \left(\frac{\frac{a+b}{2}-s}{2}\right)[u(a+b-s)-u(s)] \\
& =: B(s) \text {, }
\end{aligned}
$$

where

$B(s) \leqslant 2 L \sin \left[\frac{b-a}{8}+\frac{1}{2}\left|s-\frac{3 a+b}{4}\right|\right][u(b)-u(a)]$

$$
\begin{gathered}
\text { and } 4 L \sin \left[\frac{b-a}{8}\right] \cos \left[\frac{1}{2}\left(s-\frac{3 a+b}{4}\right)\right] \\
\times\left[\frac{u(b)-u(a)}{2}+\left|u(a+b-s)-u(s)+\frac{u(a)-u(b)}{2}\right|\right] .
\end{gathered}
$$

In particular,

$$
\begin{aligned}
& \mid f\left(\mathrm{e}^{\mathrm{i}((3 a+b) / 4)}\right)\left[u\left(\frac{a+b}{2}\right)-u(a)\right]-\int_{a}^{b} f\left(\mathrm{e}^{\mathrm{i} t}\right) \mathrm{d} u(t) \\
& \quad+f\left(\mathrm{e}^{\mathrm{i}((a+3 b) / 4)}\right)\left[u(b)-u\left(\frac{a+b}{2}\right)\right] \mid \\
& \leqslant L \int_{a}^{(a+b) / 2} \operatorname{sgn}\left(\frac{3 a+b}{4}-t\right) \cos \left(\frac{\frac{3 a+b}{4}-t}{2}\right) u(t) \mathrm{d} t \\
& \quad+L \int_{(a+b) / 2}^{b} \operatorname{sgn}\left(\frac{a+3 b}{4}-t\right) \cos \left(\frac{\frac{a+3 b}{4}-t}{2}\right) u(t) \mathrm{d} t \\
& \quad+2 L \sin \left(\frac{b-a}{8}\right)[u(b)-u(a)] \\
& =: M,
\end{aligned}
$$

where

$$
M \leqslant 2 L \sin \left(\frac{b-a}{8}\right)[u(b)-u(a)] .
$$

Proof: Since $0<b-a<2 \pi$, we have $0 \leqslant \frac{1}{2}(\mid s-$ $t \mid) \leqslant \frac{1}{2}\left(\frac{1}{2}(a+b)-a\right) \leqslant \frac{1}{2} \pi$ for any $s, t \in\left[a, \frac{1}{2}(a+b)\right]$. If $s \in\left[a, \frac{1}{2}(a+b)\right]$ and $t \in\left[\frac{1}{2}(a+b), b\right]$, then $0 \leqslant$ $\frac{1}{2}(a+b-s-t) \leqslant \frac{1}{2}\left(a+b-a-\frac{1}{2}(a+b)\right) \leqslant \frac{1}{4}(b-$ $a) \leqslant \frac{1}{2} \pi$. Hence we have $\cos \frac{1}{2}(|s-t|) \geqslant 0$ for any $s, t \in\left[a, \frac{1}{2}(a+b)\right] ; \cos \frac{1}{2}(|a+b-s-t|) \leqslant 0$ for any $s \in\left[a, \frac{1}{2}(a+b)\right], t \in\left[\frac{1}{2}(a+b), b\right]$. Using the fact that $u$ is monotonic nondecreasing on $[a, b]$,

$$
\begin{aligned}
& \int_{a}^{s} \cos \left(\frac{s-t}{2}\right) u(t) \mathrm{d} t \leqslant 2 u(s) \sin \left(\frac{s-a}{2}\right) \\
& \int_{s}^{(a+b) / 2} \cos \left(\frac{s-t}{2}\right) u(t) \mathrm{d} t \geqslant 2 u(s) \sin \left(\frac{\frac{a+b}{2}-s}{2}\right), \\
& \int_{(a+b) / 2}^{a+b-s} \cos \left(\frac{a+b-s-t}{2}\right) u(t) \mathrm{d} t \\
& \leqslant 2 u(a+b-s) \sin \left(\frac{\frac{a+b}{2}-s}{2}\right), \\
& \int_{a+b-s}^{b} \cos \left(\frac{a+b-s-t}{2}\right) u(t) \mathrm{d} t \\
& \geqslant 2 u(a+b-s) \sin \left(\frac{s-a}{2}\right) .
\end{aligned}
$$

Applying (44)-(33), we have proved (41). From the elementary property stating that

$$
\alpha x+\beta y \leqslant \max \{\alpha, \beta\}(x+y),
$$


where $\alpha, \beta, x, y \geqslant 0$, we can obtain the bounds for $B(s)$. The details are omitted.

\section{A COMPOSITE QUADRATURE RULE}

In this section, we use the results from the previous sections to approximate the Riemann-Stieltjes integral $\int_{a}^{b} f\left(\mathrm{e}^{\mathrm{i} t}\right) \mathrm{d} u(t)$, in terms of the RiemannStieltjes integral $\int_{a}^{b} f(t) \mathrm{d} u(t)$.

We consider the following partition of the interval $[a, b]$

$$
\Delta_{n}: a=x_{0}<x_{1}<\cdots<x_{n-1}<x_{n}=b
$$

and the intermediate points $\xi_{k} \in\left[x_{k}, \frac{1}{2}\left(x_{k}+x_{k+1}\right)\right]$, where $0 \leqslant k \leqslant n-1$. We define $h_{k}:=x_{k+1}-x_{k}, 0 \leqslant$ $k \leqslant n-1$ and the norm of the partition $\Delta_{n}$ is $v\left(\Delta_{n}\right)=$ $\max \left\{h_{k}: 0 \leqslant k \leqslant n-1\right\}$.

We define the quadrature rule

$$
\begin{array}{r}
O_{n}\left(f, u, \Delta_{n}, \xi\right):=\sum_{k=0}^{n-1} f\left(\mathrm{e}^{\mathrm{i} \xi_{k}}\right)\left[u\left(\frac{x_{k+1}+x_{k}}{2}\right)-u\left(x_{k}\right)\right] \\
+\sum_{k=0}^{n-1} f\left(\mathrm{e}^{\mathrm{i}\left(x_{k}+x_{k+1}-\xi_{k}\right)}\right)\left[u\left(x_{k+1}\right)-u\left(\frac{x_{k}+x_{k+1}}{2}\right)\right],
\end{array}
$$

where $f: C(0,1) \rightarrow \mathbb{C}$ is a continuous function and $u:[a, b] \subseteq[0,2 \pi] \rightarrow \mathbb{C}$ is a function of bounded variation on $[a, b]$. Define the remain$\operatorname{der} R_{n}\left(f, u, \Delta_{n}, \xi\right)$ in approximating the RiemannStieltjes integral $\int_{a}^{b} f\left(\mathrm{e}^{\mathrm{it}}\right) \mathrm{d} u(t)$ by $O_{n}\left(f, u, \Delta_{n}, \xi\right)$. Then

$$
\int_{a}^{b} f\left(\mathrm{e}^{\mathrm{i} t}\right) \mathrm{d} u(t)=O_{n}\left(f, u, \Delta_{n}, \xi\right)+R_{n}\left(f, u, \Delta_{n}, \xi\right) .
$$

We provide a priory bounds for $R_{n}\left(f, u, \Delta_{n}, \xi\right)$ in several instances of $f$ and $u$ as above in the following result.

Proposition 1 Assume that $f: C(0,1) \rightarrow \mathbb{C}$ satisfies Hölder's type condition (6). If $[a, b] \subseteq[0,2 \pi]$ and $u:[a, b] \rightarrow \mathbb{C}$ is a function of bounded variation on $[a, b]$, then for any partition $\Delta_{n}: a=x_{0}<x_{1}<\cdots<$ $x_{n-1}<x_{n}=b$ with the norm $v\left(\Delta_{n}\right) \leqslant \pi$, we have the error bound

$$
\begin{aligned}
& \left|R_{n}\left(f, u, \Delta_{n}, \xi\right)\right| \\
& \leqslant 2^{r} H \sum_{k=0}^{n-1} \sin ^{r}\left[\frac{x_{k+1}-x_{k}}{8}+\right. \\
& \left.\frac{1}{2}\left|\xi_{k}-\frac{3 x_{k}+x_{k+1}}{4}\right|\right] V_{x_{k}}^{x_{k+1}}(u)
\end{aligned}
$$

$$
\begin{aligned}
& \leqslant 2^{r} H \sum_{k=0}^{n-1} \sin ^{r}\left(\frac{x_{k+1}-x_{k}}{4}\right) V_{x_{k}}^{x_{k+1}}(u) \\
& \leqslant \frac{1}{2^{r}} H \sum_{k=0}^{n-1}\left(x_{k+1}-x_{k}\right)^{r} V_{x_{k}}^{x_{k+1}}(u) \\
& \leqslant \frac{1}{2^{r}} H v^{r}\left(\Delta_{n}\right) V_{a}^{b}(u)
\end{aligned}
$$

for any intermediate points $\xi_{k} \in\left[x_{k}, \frac{1}{2}\left(x_{k}+x_{k+1}\right)\right]$, where $0 \leqslant k \leqslant n-1$.

Proof: Since $v\left(\Delta_{n}\right) \leqslant \pi$, then on using (7) on each interval $\left[x_{k}, x_{k+1}\right]$ and for any intermediate points $\xi_{k} \in\left[x_{k}, \frac{1}{2}\left(x_{k}+x_{k+1}\right)\right]$ where $0 \leqslant k \leqslant n-1$, we have

$$
\begin{aligned}
& \mid f\left(\mathrm{e}^{\mathrm{i} \xi_{k}}\right)\left[u\left(\frac{x_{k}+x_{k+1}}{2}\right)-u\left(x_{k}\right)\right]+ \\
& \quad f\left(\mathrm{e}^{\mathrm{i}\left(x_{k}+x_{k+1}-\xi_{k}\right)}\right)\left[u\left(x_{k+1}\right)-u\left(\frac{x_{k}+x_{k+1}}{2}\right)\right] \\
& \quad-\int_{x_{k}}^{x_{k+1}} f\left(\mathrm{e}^{\mathrm{i} t}\right) \mathrm{d} u(t) \mid \\
& \leqslant 2^{r} H \sin ^{r}\left[\frac{x_{k+1}-x_{k}}{8}+\right. \\
& \left.\quad \frac{1}{2}\left|\xi_{k}-\frac{3 x_{k}+x_{k+1}}{4}\right|\right] V_{x_{k}}^{x_{k+1}}(u) \\
& \leqslant 2^{r} H \sin ^{r}\left(\frac{x_{k+1}-x_{k}}{4}\right) V_{x_{k}}^{x_{k+1}}(u) \\
& \leqslant \frac{1}{2^{r}} H\left(x_{k+1}-x_{k}\right)^{r} V_{x_{k}}^{x_{k+1}}(u) .
\end{aligned}
$$

Summing over $k$ from 0 to $n-1$ in (48) and using the generalized Delta inequality, we deduce (47).

Remark 7 If we choose $\xi_{k}=\frac{1}{2}\left(\lambda_{k}+\lambda_{k+1}\right)$, we recapture Corollary 1 of Ref. 3. In particular, if we choose $\xi_{k}=\left(3 x_{k}+x_{k+1}\right) / 4$, then we obtain $\left|R_{n}\left(f, u, \Delta_{n}, \xi\right)\right| \leqslant\left(1 / 4^{r}\right) H v^{r}\left(\Delta_{n}\right) V_{a}^{b}(u)$, which is more precise than Proposition 4 of Ref. 3.

Corollary 3 Under the assumption of Proposition 1 and $\xi_{k}=\left(3 x_{k}+x_{k+1}\right) / 4$, we define the special quadrature rule by

$$
\begin{aligned}
& T_{n}\left(f, u, \Delta_{n}\right) \\
& \quad:=\sum_{k=0}^{n-1} f\left(\mathrm{e}^{\mathrm{i}\left(\left(3 x_{k}+x_{k+1}\right) / 4\right)}\right)\left[u\left(\frac{x_{k}+x_{k+1}}{2}\right)-u\left(x_{k}\right)\right] \\
& +\sum_{k=0}^{n-1} f\left(\mathrm{e}^{\mathrm{i}\left(\left(x_{k}+3 x_{k+1}\right) / 4\right)}\right)\left[u\left(x_{k+1}\right)-u\left(\frac{x_{k}+x_{k+1}}{2}\right)\right]
\end{aligned}
$$


and the error $E_{n}\left(f, u, \Delta_{n}\right)$ by

$$
\int_{a}^{b} f\left(\mathrm{e}^{\mathrm{i} t}\right) \mathrm{d} u(t)=T_{n}\left(d, u, \Delta_{n}\right)+E_{n}\left(f, u, \Delta_{n}\right) .
$$

Then we have the error bounds

$$
\begin{aligned}
& \left|E_{n}\left(f, u, \Delta_{n}\right)\right| \\
& \quad \leqslant 2^{r} H \sum_{k=0}^{n-1} \sin ^{r}\left(\frac{x_{k+1}-x_{k}}{8}\right) V_{x_{k}}^{x_{k+1}}(u) \\
& \quad \leqslant \frac{1}{4^{r}} H \sum_{k=0}^{n-1}\left(x_{k+1}-x_{k}\right)^{r} V_{x_{k}}^{x_{k+1}}(u) \\
& \quad \leqslant \frac{1}{4^{r}} H v^{r}\left(\Delta_{n}\right) V_{a}^{b}(u) .
\end{aligned}
$$

In the following result, we consider the case of both integrator and integrand being Lipschitzian.

Proposition 2 Under the assumption of Theorem 3, for any partition $\Delta_{n}: a=x_{0}<x_{1}<\cdots<x_{n-1}<$ $x_{n}=b$, we have the error bound

$$
\begin{aligned}
& \left|E_{n}\left(f, u, \Delta_{n}, \xi\right)\right| \\
& \quad \leqslant 16 K L \sum_{k=0}^{n-1}\left[\sin ^{2}\left(\frac{\xi_{k}-x_{k}}{4}\right)+\sin ^{2}\left(\frac{\frac{x_{k}+x_{k+1}}{2}-\xi_{k}}{4}\right)\right] \\
& \quad \leqslant 32 K L \sum_{k=0}^{n-1} \sin ^{2}\left(\frac{x_{k+1}-x_{k}}{8}\right) \\
& \quad \leqslant \frac{1}{2} K L \sum_{k=0}^{n-1}\left(x_{k+1}-x_{k}\right)^{2} \\
& \quad \leqslant \frac{1}{2} K L(b-a) v\left(\Delta_{n}\right)
\end{aligned}
$$

for any $\xi_{k} \in\left[x_{k}, \frac{1}{2}\left(x_{k}+x_{k+1}\right)\right]$, where $0 \leqslant k \leqslant n-1$. In particular,

$$
\begin{aligned}
& \left|E_{n}\left(f, u, \Delta_{n}\right)\right| \\
& \quad \leqslant 32 K L \sum_{k=0}^{n-1} \sin ^{2}\left(\frac{x_{k+1}-x_{k}}{16}\right) \\
& \quad \leqslant \frac{1}{8} K L \sum_{k=0}^{n-1}\left(x_{k+1}-x_{k}\right)^{2} \\
& \quad \leqslant \frac{1}{8} K L(b-a) v\left(\Delta_{n}\right) .
\end{aligned}
$$

The proof is similar to Theorem 3 and the details are omitted.

Proposition 3 Under the assumption of Theorem 4, for any quadrature $\Delta_{n}: a=x_{0}<x_{1}<\cdots<x_{n-1}<$ $x_{n}=b$ with the norm $v\left(\Delta_{n}\right) \leqslant \pi$ and $\xi_{k} \in\left[x_{k}, \frac{1}{2}\left(x_{k}+\right.\right.$ $\left.x_{k+1}\right)$, we have the error bound

$\left|R_{n}\left(f, u, \Delta_{n}, \xi\right)\right|$

$$
\begin{aligned}
& \leqslant 2 L \sum_{k=0}^{n-1} \sin \left(\frac{\xi_{k}-x_{k}}{2}\right)\left[u\left(x_{k+1}\right)-u\left(x_{k}\right)\right] \\
& +L \sum_{k=0}^{n-1} \int_{x_{k}}^{\left(x_{k}+x_{k+1}\right) / 2} \operatorname{sgn}\left(\xi_{k}-t\right) \cos \left(\frac{\xi_{k}-t}{2}\right) u(t) \mathrm{d} t \\
& +L \sum_{k=0}^{n-1} \int_{\left(x_{k}+x_{k+1}\right) / 2}^{x_{k+1}} \operatorname{sgn}\left(x_{k}+x_{k+1}-\xi_{k}-t\right) \\
& \times \cos \left(\frac{x_{k}+x_{k+1}-\xi_{k}-t}{2}\right) u(t) \mathrm{d} t \\
& \leqslant 2 L \sum_{k=0}^{n-1}\left[\operatorname { s i n } ( \frac { \xi _ { k } - x _ { k } } { 2 } ) \left[u\left(x_{k+1}\right)-u\left(x_{k}\right)+u\left(\xi_{k}\right)\right.\right. \\
& \left.\left.-u\left(x_{k}+x_{k+1}-\xi_{k}\right)\right]\right]+2 L \sum_{k=0}^{n-1}\left[\operatorname { s i n } \left(\frac{x_{k}+x_{k+1}}{4}\right.\right. \\
& \left.\left.-\frac{\xi_{k}}{2}\right)\left[u\left(x_{k}+x_{k+1}-\xi_{k}\right)-u\left(\xi_{k}\right)\right]\right] \\
& \leqslant 2 L \sum_{k=0}^{n-1} \sin \left[\frac{x_{k+1}-x_{k}}{8}\right. \\
& \left.+\frac{1}{2}\left|\xi_{k}-\frac{3 x_{k}+x_{k+1}}{4}\right|\right]\left[u\left(x_{k+1}\right)-u\left(x_{k}\right)\right] \\
& \leqslant 2 L \sum_{k=0}^{n-1} \sin \left(\frac{x_{k+1}-x_{k}}{4}\right)\left[u\left(x_{k+1}\right)-u\left(x_{k}\right)\right] \\
& \leqslant \frac{L}{2} \sum_{k=0}^{n-1}\left(x_{k+1}-x_{k}\right)\left[u\left(x_{k+1}\right)-u\left(x_{k}\right)\right] \\
& \leqslant \frac{\Delta_{n} L}{2}[u(b)-u(a)]
\end{aligned}
$$

where $0 \leqslant k \leqslant n-1$. In particular,

$$
\begin{aligned}
& \left|E_{n}\left(f, u, \Delta_{n}\right)\right| \\
& \quad \leqslant 2 L \sum_{k=0}^{n-1} \sin \left(\frac{x_{k+1}-x_{k}}{8}\right)\left[u\left(x_{k+1}\right)-u\left(x_{k}\right)\right] \\
& \quad \leqslant \frac{L}{4} \sum_{k=0}^{n-1}\left(x_{k+1}-x_{k}\right)\left[u\left(x_{k+1}\right)-u\left(x_{k}\right)\right] \\
& \quad \leqslant \frac{L}{4} v\left(\Delta_{n}\right)[u(b)-u(a)] .
\end{aligned}
$$

The proof is similar to Corollary 2 and details are omitted.

Remark 8 If we choose $\xi_{k}=\frac{1}{2}\left(\lambda_{k}+\lambda_{k+1}\right)$, we recapture Proposition 3 of Ref. 3 . If we choose $\xi_{k}=\left(3 \lambda_{k}+\lambda_{k+1}\right) / 4$, we have $\left|E_{n}\left(f, u, \Delta_{n}\right)\right| \leqslant$ $\frac{1}{4} L v\left(\Delta_{n}\right)[u(b)-u(a)]$, which is more precise than Proposition 3 of Ref. 3. 


\section{APPLICATIONS FOR FUNCTIONS OF UNITARY OPERATORS}

In Ref. 3, the author used inequality (3) to give estimates of a unitary operator. In this section, we apply our previous inequality (7) to give estimates of unitary operators $U$ defined on complex Hilbert spaces.

We recall here some basic facts on unitary operators and spectral families. We say that the bounded linear operator $U: H \rightarrow H$ on the Hilbert space $H$ is unitary if $U^{*}=U^{-1}$.

It is well known that if $U$ is a unitary operator, there exists a family of projections $\left\{E_{\lambda}\right\}_{\lambda \in[0,2 \pi]}$, called the spectral family of $U$ with the following properties ${ }^{20}$ :

(i) $E_{\lambda} \leqslant E_{\mu}$ for $0 \leqslant \lambda \leqslant \mu \leqslant 2 \pi$;

(ii) $E_{0}=0$ and $E_{2 \pi}=1_{H}$ (the identity operator on $H$ );

(iii) $E_{\lambda+0}=E_{\lambda}$ for $0 \leqslant \lambda<2 \pi$;

(iv) $U=\int_{0}^{2 \pi} \mathrm{e}^{\mathrm{i} \lambda} \mathrm{d} E_{\lambda}$, where the integral is of Riemann-Stieltjes type.

Furthermore, if $\left\{F_{\lambda}\right\}_{\lambda \in[0,2 \pi]}$ is a family of projections satisfying these requirements for the operator $U$, then $F_{\lambda}=E_{\lambda}$ for all $\lambda \in[0,2 \pi]$.

Also, for every continuous complex valued function $f: C(0,1) \rightarrow \mathbb{C}$ on the complex unit circle $C(0,1)$, we have

$$
f(U)=\int_{0}^{2 \pi} f\left(\mathrm{e}^{\mathrm{i} \lambda}\right) \mathrm{d} E_{\lambda},
$$

where the integral is taken in the Riemann-Stieltjes sense. In particular, we have

$$
\langle f(U) x, y\rangle=\int_{0}^{2 \pi} f\left(\mathrm{e}^{\mathrm{i} \lambda}\right) \mathrm{d}\left\langle E_{\lambda} x, y\right\rangle
$$

and

$$
\begin{aligned}
\|f(U) x\|^{2} & =\int_{0}^{2 \pi}\left|f\left(\mathrm{e}^{\mathrm{i} \lambda}\right)\right|^{2} \mathrm{~d}\left\|E_{\lambda} x\right\|^{2} \\
& =\int_{0}^{2 \pi}\left|f\left(\mathrm{e}^{\mathrm{i} \lambda}\right)\right|^{2} \mathrm{~d}\left\langle E_{\lambda} x, x\right\rangle,
\end{aligned}
$$

for any vector $x, y \in H$. We consider the following partition of the interval $[0,2 \pi]$ :

$$
\Delta_{n}: 0=\lambda_{0}<\lambda_{1}<\cdots<\lambda_{n-1}<\lambda_{n}=2 \pi
$$

and the intermediate points $\xi_{k} \in\left[\lambda_{k}, \frac{1}{2}\left(\lambda_{k}+\lambda_{k+1}\right)\right]$, where $0 \leqslant k \leqslant n-1$. We define $h_{k}:=\lambda_{k+1}-\lambda_{k}$, $0 \leqslant k \leqslant n-1$ and the norm of the partition $\Delta_{n}$ is $v\left(\Delta_{n}\right)=\max \left\{h_{k}: 0 \leqslant k \leqslant n-1\right\}$.
If $U$ is a unitary operator on the Hilbert space $H$ and $\left\{E_{\lambda}\right\}_{\lambda \in[0,2 \pi]}$ is the spectral family of $U$, then

$$
\begin{aligned}
& O_{n}\left(f, U, \Delta_{n}, \xi ; x, y\right) \\
& \quad:=\sum_{k=0}^{n-1} f\left(\mathrm{e}^{\mathrm{i} \xi_{k}}\right)\left\langle\left(E_{\left(\lambda_{k}+\lambda_{k+1}\right) / 2}-E_{\lambda_{k}}\right) x, y\right\rangle \\
& +\sum_{k=0}^{n-1} f\left(\mathrm{e}^{\mathrm{i}\left(x_{k+1}+x_{k}-\xi_{k}\right)}\right)\left\langle\left(E_{\lambda_{k+1}}-E_{\left(\lambda_{k}+\lambda_{k+1}\right) / 2}\right) x, y\right\rangle
\end{aligned}
$$

and

$$
\begin{aligned}
& T_{n}\left(f, U, \Delta_{n} ; x, y\right) \\
& :=\sum_{k=0}^{n-1} f\left(\mathrm{e}^{\mathrm{i}\left(3 \lambda_{k}+\lambda_{k+1}\right) / 4}\right)\left\langle\left(E_{\left(\lambda_{k}+\lambda_{k+1}\right) / 2}-E_{\lambda_{k}}\right) x, y\right\rangle \\
& +\sum_{k=0}^{n-1} f\left(\mathrm{e}^{\mathrm{i}\left(\lambda_{k}+3 \lambda_{k+1}\right) / 4}\right)\left\langle\left(E_{\lambda_{k+1}}-E_{\left(\lambda_{k}+\lambda_{k+1}\right) / 2}\right) x, y\right\rangle,
\end{aligned}
$$

where $x, y \in H$.

Theorem 5 With the above assumptions for $U$, $\left\{E_{\lambda}\right\}_{\lambda \in[0,2 \pi]}, \Delta_{n}$ with $v\left(\Delta_{n}\right) \leqslant \pi$ and if $f: C(0,1)$ $\rightarrow \mathbb{C}$ satisfies Hölder's type condition (6), then we have the representation

$$
\begin{aligned}
\langle f(U) x, y\rangle=O_{n}\left(f, U, \Delta_{n}, \xi ; x, y\right) & \\
& +R_{n}\left(f, U, \Delta_{n}, \xi ; x, y\right)
\end{aligned}
$$

with the error $R_{n}\left(f, U, \Delta_{n}, \xi ; x, y\right)$ which satisfies the bounds

$$
\begin{aligned}
& \left|R_{n}\left(f, U, \Delta_{n}, \xi ; x, y\right)\right| \\
& \quad \leqslant 2^{r} H \sum_{k=0}^{n-1} \sin ^{r}\left[\frac{\lambda_{k+1}-\lambda_{k}}{8}+\right. \\
& \left.\quad \frac{1}{2}\left|\xi_{k}-\frac{3 \lambda_{k}+\lambda_{k+1}}{4}\right|\right] V_{\lambda_{k}}^{\lambda_{k+1}\left(\left\langle E_{(\cdot)} x, y\right\rangle\right)} \\
& \leqslant \frac{1}{2^{r}} H \sum_{k=0}^{n-1}\left(\lambda_{k+1}-\lambda_{k}\right)^{r} V_{\lambda_{k}}^{\lambda_{k+1}}\left(\left\langle E_{(\cdot)} x, y\right\rangle\right) \\
& \leqslant \frac{H}{2^{r}} v^{r}\left(\Delta_{n}\right)\|x\|\|y\|,
\end{aligned}
$$

for any $x, y \in H$ and the intermediate points $\xi_{k} \in$ $\left[\lambda_{k}, \frac{1}{2}\left(\lambda_{k}+\lambda_{k+1}\right)\right]$, where $0 \leqslant k \leqslant n-1$. In particular, we have

$\langle f(U) x, y\rangle=T_{n}\left(f, U, \Delta_{n} ; x, y\right)+E_{n}\left(f, U, \Delta_{n} ; x, y\right)$, 
with the error

$$
\begin{aligned}
& \left|E_{n}\left(f, U, \Delta_{n} ; x, y\right)\right| \\
& \quad \leqslant 2^{r} H \sum_{k=0}^{n-1} \sin ^{r}\left(\frac{\lambda_{k+1}-\lambda_{k}}{8}\right) V_{\lambda_{k}}^{\lambda_{k+1}}\left(\left\langle E_{(\cdot)} x, y\right\rangle\right) \\
& \quad \leqslant \frac{H}{4^{r}} \nu^{r}\left(\Delta_{n}\right)\|x\|\|y\|,
\end{aligned}
$$

for any vector $x, y \in H$.

Proof: For any $x, y \in H$, we define $u(\lambda):=\left\langle E_{\lambda} x, y\right\rangle$, $\lambda \in[0,2 \pi]$. We know that $u$ is of bounded variation, and from Ref. 3,

$$
V_{0}^{2 \pi}(u)=: V_{0}^{2 \pi}\left(\left\langle E_{(\cdot)} x, y\right\rangle\right) \leqslant\|x\|\|y\| .
$$

By (7) and (65), (62) can be proved.

Remark 9 If we choose $\xi_{k}=\frac{1}{2}\left(\lambda_{k}+\lambda_{k+1}\right)$, we recapture Theorem 6 of Ref. 3 . If we choose $\xi_{k}=\left(3 \lambda_{k}+\lambda_{k+1}\right) / 4$, we have $\left|E_{n}\left(f, U, \Delta_{n} ; x, y\right)\right| \leqslant$ $\left(H / 4^{r}\right) v^{r}\left(\Delta_{n}\right)\|x\|\|y\|$, which is more precise than Theorem 6 of Ref. 3.

Remark 10 In the case when the partition reduces to the whole interval $[0,2 \pi]$, then making use of (7), for any $s \in[0, \pi]$ and any vectors $x, y \in H$, we have the bound

$$
\begin{aligned}
& \mid f\left(\mathrm{e}^{\mathrm{i} s}\right)\left\langle E_{\pi} x, y\right\rangle+ f\left(\mathrm{e}^{\mathrm{i}(2 \pi-s)}\right)\left\langle\left(1_{H}-E_{\pi}\right) x, y\right\rangle \\
&-\langle f(U) x, y\rangle \mid \\
& \leqslant 2^{r} H \sin ^{r}\left[\frac{\pi}{4}+\frac{1}{2}\left|s-\frac{\pi}{2}\right|\right] V_{0}^{2 \pi}\left(\left\langle E_{(\cdot)} x, y\right\rangle\right) .
\end{aligned}
$$

If we obtain $s=\frac{1}{2} \pi$, we obtain the best inequality

$$
\begin{gathered}
\mid f(\mathrm{i})\left\langle E_{\pi} x, y\right\rangle+f(-\mathrm{i})\left\langle\left(1_{H}-E_{\pi}\right) x, y\right\rangle \\
-\langle f(U) x, y\rangle \mid \\
\leqslant 2^{r / 2} H V_{0}^{2 \pi}\left(\left\langle E_{(\cdot)} x, y\right\rangle\right) \leqslant 2^{r / 2} H\|x\|\|y\|,
\end{gathered}
$$

for any vectors $x, y \in H$.

If $U$ is a unitary operator on the Hilbert space $H$ and $\left\{E_{\lambda}\right\}_{\lambda \in[0,2 \pi]}$ is the spectral family of $U$. Depending only one vector $x \in H$, we can introduce the following sums

$$
\begin{gathered}
\tilde{O}_{n}\left(f, U, \Delta_{n}, \xi ; x\right) \\
:=\sum_{k=0}^{n-1} f\left(\mathrm{e}^{\mathrm{i} \xi_{k}}\right)\left\langle\left(E_{\left(\lambda_{k}+\lambda_{k+1}\right) / 2}-E_{\lambda_{k}}\right) x, x\right\rangle \\
+\sum_{k=0}^{n-1} f\left(\mathrm{e}^{\mathrm{i}\left(\lambda_{k}+\lambda_{k+1}-\xi_{k}\right)}\right)\left\langle\left(E_{\lambda_{k+1}}-E_{\left(\lambda_{k}+\lambda_{k+1}\right) / 2}\right) x, x\right\rangle .
\end{gathered}
$$

Theorem 6 If $f: C(0,1) \rightarrow \mathbb{C}$ is Lipschitzian with the constant $L>0$ on the circle $C(0,1), \Delta_{n}$ with $v\left(\Delta_{n}\right) \leqslant \pi$ and $U,\left\{E_{\lambda}\right\}_{\lambda \in[0,2 \pi]}$ are defined above, then we have the representation

$$
\langle f(U) x, x\rangle=\tilde{O}_{n}\left(f, U, \Delta_{n}, \xi ; x\right)+\tilde{R}_{n}\left(f, U, \Delta_{n}, \xi ; x\right)
$$

with the error $\tilde{R}_{n}\left(f, U, \Delta_{n}, \xi ; x\right)$ which satisfies the bounds

$$
\begin{aligned}
& \left|\tilde{R}_{n}\left(f, U, \Delta_{n}, \xi ; x\right)\right| \\
& \quad \leqslant 2 L \sum_{k=0}^{n-1} \sin \left[\frac{\lambda_{k+1}-\lambda_{k}}{8}+\right. \\
& \left.\quad \frac{1}{2}\left|\xi_{k}-\frac{3 \lambda_{k}+\lambda_{k+1}}{4}\right|\right]\left\langle\left(E_{\lambda_{k+1}}-E_{\lambda_{k}}\right) x, x\right\rangle \\
& \quad \leqslant \frac{v\left(\Delta_{n}\right)}{2} L\|x\|^{2},
\end{aligned}
$$

for any vectors $x \in H$ and the intermediate points $\xi_{k} \in$ $\left[\lambda_{k}, \frac{1}{2}\left(\lambda_{k}+\lambda_{k+1}\right)\right]$, where $0 \leqslant k \leqslant n-1$. In particular, we have

$$
\langle f(U) x, x\rangle=\tilde{T}_{n}\left(f, U, \Delta_{n} ; x\right)+\tilde{E}_{n}\left(f, U, \Delta_{n} ; x\right)
$$

with the error

$$
\begin{aligned}
& \left|\tilde{E}_{n}\left(f, U, \Delta_{n} ; x\right)\right| \\
& \quad \leqslant \frac{1}{4} L \sum_{k=0}^{n-1}\left(\lambda_{k+1}-\lambda_{k}\right)\left\langle\left(E_{\lambda_{k+1}}-E_{\lambda_{k}}\right) x, x\right\rangle \\
& \quad \leqslant \frac{1}{4} \operatorname{Lv}\left(\Delta_{n}\right)\|x\|^{2},
\end{aligned}
$$

for any $x \in H$.

Proof: The proof follows from Proposition 3 applied for the monotonic nondecreasing function $u(t):=$ $\left\langle E_{t} x, x\right\rangle, t \in[0,2 \pi]$.

Remark 11 If we choose $\xi_{k}=\frac{1}{2}\left(\lambda_{k}+\lambda_{k+1}\right)$, we recapture Theorem 7 of Ref. 3 . If we choose $\xi_{k}=\left(3 \lambda_{k}+\lambda_{k+1}\right) / 4$, we have $\left|\tilde{E}_{n}\left(f, U, \Delta_{n} ; x\right)\right| \leqslant$ $\frac{1}{4} L v\left(\Delta_{n}\right)\|x\|^{2}$, which is more precise than Theorem 7 of Ref. 3.

Remark 12 In the case when the partition reduces to the whole interval $[0,2 \pi]$, then by the above result, we obtain

$$
\begin{aligned}
& \mid f\left(\mathrm{e}^{\mathrm{i} s}\right)\left\langle E_{\pi} x, x\right\rangle+ f\left(\mathrm{e}^{\mathrm{i}(2 \pi-s)}\right)\left\langle\left(1_{H}-E_{\pi}\right) x, x\right\rangle \\
&-\langle f(U) x, x\rangle \mid
\end{aligned}
$$




$$
\begin{array}{r}
\leqslant L \int_{0}^{\pi} \operatorname{sgn}(s-t) \cos \left(\frac{s-t}{2}\right)\left\langle E_{t} x, x\right\rangle \mathrm{d} t \\
+L \int_{\pi}^{2 \pi} \operatorname{sgn}(2 \pi-s-t) \cos \left(\frac{2 \pi-s-t}{2}\right)\left\langle E_{t} x, x\right\rangle \mathrm{d} t \\
+2 L \sin \left(\frac{s}{2}\right)\|x\|^{2}, \quad \text { (73) }
\end{array}
$$

for any $s \in[0, \pi]$ and $x \in H$.

Example 1 We choose two complex functions as follows to provide some simple examples for the inequalities above.

(a) Consider the power function $f: C \backslash\{0\} \rightarrow$ $\mathbb{C}, f(z)=z^{m}$ where $m$ is a non-zero integer. Then, clearly, for any $z, w$ belonging to the unit circle $C(0,1)$, we have the inequality

$$
|f(z)-f(w)| \leqslant|m||z-w|,
$$

which shows that $f$ is Lipschitzian with the constant $L=|m|$ on the circle $C(0,1)$. Then from (63), for any unitary operator $U$, we obtain

$$
\begin{aligned}
& \left|\mathrm{e}^{\mathrm{i} m s}\left\langle E_{\pi} x, y\right\rangle+\mathrm{e}^{\mathrm{i} m(2 \pi-s)}\left\langle\left(1_{H}-E_{\pi}\right) x, y\right\rangle-\left\langle U^{m} x, y\right\rangle\right| \\
& \quad \leqslant 2|m| \sin \left[\frac{\pi}{4}+\frac{1}{2}\left|s-\frac{\pi}{2}\right|\right] V_{0}^{2 \pi}\left(\left\langle E_{(\cdot)} x, y\right\rangle\right), \quad \text { (74) }
\end{aligned}
$$

for any vectors $x, y \in H$, where $\left\{E_{\lambda}\right\}_{\lambda \in[0,2 \pi]}$ is the spectral family of $U$. If we obtain $s=\frac{1}{2} \pi$, then the best inequality is

$$
\begin{aligned}
& \left|\mathrm{i}^{m}\left\langle E_{\pi} x, y\right\rangle+(-\mathrm{i})^{m}\left\langle\left(1_{H}-E_{\pi}\right) x, y\right\rangle-\left\langle U^{m} x, y\right\rangle\right| \\
& \leqslant \sqrt{2}|m| V_{0}^{2 \pi}\left(\left\langle E_{(\cdot)} x, y\right\rangle\right) \leqslant \sqrt{2}|m|\|x\|\|y\|,
\end{aligned}
$$

for any vectors $x, y \in H$.

(b) For $a \neq \pm 1,0$, consider the function $f$ : $C(0,1) \rightarrow \mathbb{C}, f_{a}(z)=1 /(1-a z)$. From Ref. 3, we have

$$
\left|f_{a}(z)-f_{a}(w)\right| \leqslant \frac{|a|}{(1-|a|)^{2}}|z-w|,
$$

for any $z, w \in C(0,1)$, showing that the function $f_{a}$ is Lipschitzian with the constant $L_{a}=|a| /(1-|a|)^{2}$ on the circle $C(0,1)$. Then from (63), for any unitary operator $U$, we obtain

$$
\begin{aligned}
\mid\left(1-a \mathrm{e}^{\mathrm{i} s}\right)^{-1}\left\langle E_{\pi} x, y\right\rangle-\left\langle\left(1_{H}-a U\right)^{-1} x, y\right\rangle & \\
& +\left(1-a \mathrm{e}^{\mathrm{i}(2 \pi-s)}\right)^{-1}\left\langle\left(1_{H}-E_{\pi}\right) x, y\right\rangle \mid \\
\leqslant & \frac{2|a|}{(1-|a|)^{2}} \sin \left[\frac{\pi}{4}+\frac{1}{2}\left|s-\frac{\pi}{2}\right|\right] V_{0}^{2 \pi}\left(\left\langle E_{(\cdot)} x, y\right\rangle\right),
\end{aligned}
$$

for any vectors $x, y \in H$, where $\left\{E_{\lambda}\right\}_{\lambda \in[0,2 \pi]}$ is the spectral family of $U$. If we obtain $s=\frac{1}{2} \pi$, then the best inequality is

$$
\begin{gathered}
\mid(1-a \mathrm{i})^{-1}\left\langle E_{\pi} x, y\right\rangle-\left\langle\left(1_{H}-a U\right)^{-1} x, y\right\rangle \\
+(1+a \mathrm{i})^{-1}\left\langle\left(1_{H}-E_{\pi}\right) x, y\right\rangle \mid \\
\leqslant \frac{\sqrt{2}|a|}{(1-|a|)^{2}} V_{0}^{2 \pi}\left(\left\langle E_{(\cdot)} x, y\right\rangle\right) \leqslant \frac{\sqrt{2}|a|}{(1-|a|)^{2}}\|x\|\|y\|,
\end{gathered}
$$

for any vectors $x, y \in H$.

\section{REFERENCES}

1. Alomari MW (2013) A companion of Ostrowski's inequality for the Riemann-Stieltjes integral $\int_{a}^{b} f(t) d u(t)$, where $f$ is of $r-H$-Hölder type and $u$ is of bounded variation and applications. Appl Math Comput 9, 4792-9.

2. Dragomir SS (2014) A companion of Ostrowski's inequality for functions of bounded variation and applications. Int J Nonlin Anal Appl 5, 89-97.

3. Dragomir SS (2015) Ostrowski's type inequalities for complex functions defined on unit circle with applications for unitary operators in Hilbert spaces. Arch Math 51, 233-54.

4. Anastassiou GA (2007) Grüss type inequalities for the Stieltjes integral. Nonlin Funct Anal Appl 12, 583-93.

5. Alomari MW (2012) Some Grüss type inequalities for Riemann-Stieltjes integral and applications. Acta Math Univ Comenian 81, 211-20.

6. Barnett NS, Cheung W-S, Dragomir SS, Sofo A (2009) Ostrowski and trapezoid type inequalities for the Stieltjes integral with Lipschitzian integrands or integrators. Comput Math Appl 57, 195-201.

7. Budak H, Sarikaya MZ (2015) A companion of generalization of Ostrowski type inequalities for functions of two variables with bounded variation. www. researchgate.net/publication/279874402.

8. Budak H, Sarikaya MZ (2017) A companion of Ostrowski type inequalities for mappings of bounded variation and some applications. Trans A Razmadze Math Inst 171, 136-43.

9. Cerone P, Cheung WS, Dragomir SS (2007) On Ostrowski type inequalities for Stieltjes integrals with absolutely continuous integrands and integrators of bounded variation. Comput Math Appl 54, 183-91.

10. Cheung WS, Dragomir SS (2007) Two Ostrowski type inequalities for the Stieltjes integral of monotonic functions. Bull Aust Math Soc 75, 299-311.

11. Cerone P, Dragomir SS, Roumeliotis J (1999) An inequality of Ostrowski-Grüss type for twice differentiable mappings and applications in numerical integration. Kyungpook Math J 39, 333-41. 
12. Dragomir SS (2015) Generalised trapezoid-type inequalities for complex functions defined on unit circle with applications for unitary operators in Hilbert spaces. Mediterr J Math 12, 573-91.

13. Dragomir SS (2016) Trapezoid type inequalities for complex functions defined on the unit circle with applications for unitary operators in Hilbert spaces. Georgian Math J 23, 199-210.

14. Dragomir SS (2011) Ostrowski's type inequalities for some classes of continuous functions of selfadjoint operators in Hilbert spaces. Comput Math Appl 62, 4439-48.

15. Dragomir SS (2012) Operator Inequalities of Ostrowski and Trapezoidal Type, Springer, New York.

16. Dragomir SS, Rassias TM (2002) Ostrowski Type Inequalities and Applications in Numerical Integration, Kluwer, Dordrecht.

17. Liu W, Sun Y (2012) A refinement of the companion of Ostrowski inequality for functions of bounded variation and applications. arXiv:1207.3861 [math.FA].

18. Qayyum A, Shoaib M, Faye I (2016) Companion of Ostrowski-type inequality based on 5-step quadratic kernel and applications. J Nonlin Sci Appl 9, 537-52.

19. Sarikaya MZ, Budak H, Qayyum A (2016) An improved version of perturbed companion of Ostrowski type inequalities. $J$ Inequal Spec Functions 7, 12-25.

20. Helmberg G (1969) Introduction to Spectral Theory in Hilbert Space, North-Holland. 